\title{
Lineamientos para la educación rural en el posconflicto: Marco del acuerdo de paz Colombia 2016
}

\author{
Post conflict guidelines for rural education in \\ the framework of peace agreement - Colombia 2016
}

Luis Daniel Vargas Sánchez $z^{2}$

\section{Resumen}

Después de más de cincuenta años de confrontación armada, el Gobierno de Colombia y las Fuerzas Armadas Revolucionarias de Colombia-FARC, luego de un largo proceso de paz, llegaron a firmar "El Acuerdo Final para la Terminación del Conflicto y la Construcción de una Paz Estable y Duradera" (2016), en donde el Estado debe establecer Planes Nacionales para la prestación de varios servicios públicos esenciales, entre ellos la educación, proceso analizado en la investigación sistematizada en este artículo. De esta manera, en el contexto socioeconómico de la Región Caribe y la Nación, se analizan indicadores de pobreza, desempleo, generación de ingresos, capital humano, analfabetismo, cobertura y calidad educativa, frente a las concepciones normativas de la Educación Rural y los diferentes modelos de educación flexibles implementados en el sector rural. Finalmente, se analiza el desarrollo de los bienes y servicios de las Tecnologías de la Información y la Comunicación-TIC en los establecimientos educativos y su impacto en la educación rural, terminando con unas recomendaciones producto de investigaciones y experiencias administrativas.

Palabras clave: Acuerdo de Paz; Educación rural; Enfoque territorial; Desarrollo social.

\begin{abstract}
After more than fifty years of armed confrontation, the Government of Colombia and the Revolutionary Armed Forces of Colombia-FARC, after a long p eace process, signed "The Final Agreement for the Completion of Conflict and the Construction of a Peace Stable and Durable "(2016), here it is pointed out that National Plans promoted by the State should be established for the provision of several essential public services, including education, a process analyzed in the research which is systematized in this paper. Thus, in the socio-economic context of the Caribbean Region and the Nation, indicators of poverty, unemployment, income generation, human capital, illiteracy, coverage and educational quality are analyzed, as opposed to the normative conceptions of Rural Education and the different Flexible education models implemented in the rural sector. Finally, the development of ICT Information and Communication Technologies (ICT) goods and services in educational establishments and their impact on rural education are analized, ending with recommendations based on research and administrative experiences.
\end{abstract}

Keywords: Peace Agreement; Rural education; Territorial approach; Social development.

${ }^{2}$ Magister en Educación - Universidad de Cartagena-SUE Caribe. E.mail: luisdvargas07@gmail.com 


\section{Introducción}

El Acuerdo Final para la Terminación del Conflicto y la Construcción de una Paz Estable y Duradera, suscrito entre el Gobierno de Colombia y la guerrilla de las FARC (2016), se ocupa de manera directa del sector de la educación, razón para entrar a analizar los contenidos y alcances de estos temas, en especial en el área rural desde la investigación con el rigor científico y la pertinencia social.

Desde la Constitución colombiana se definen los conceptos y relaciones con la educación cuando se expresa: "La educación es un derecho de la persona y un servicio público que tiene una función social; con ella se busca el acceso al conocimiento, a la ciencia, a la técnica, y a los demás bienes y valores de la cultura (...). La educación formará al colombiano en el respeto a los derechos humanos, a la paz y a la democracia y en la práctica del trabajo y la recreación, para el mejoramiento cultural, científico, tecnológico y para la protección del ambiente (...). El Estado, la sociedad y la familia son responsables de la educación" (...), son los postulados que trae el artículo 67 de la Constitución Política de Colombia (1991) y que tocan en esencia los principios y valores de toda sociedad civilizada.

Desde hace décadas, los nacidos y crecidos en medio del conflicto armado de Colombia, han escuchado y leído que la guerra se lleva los recursos de la educación y que siempre, el presupuesto de la guerra fue mayor al de la educación hasta la vigencia pasada (2016).

El Acuerdo Final de Paz (2016) en relación con la educación inicia "Subrayando que el Acuerdo Final presta especial atención a los derechos fundamentales de las mujeres, los de los grupos sociales vulnerables como son los pueblos indígenas, las niñas, niños y adolescentes, las comunidades afro descendientes y otros grupos étnicamente diferenciados; los derechos fundamentales de los campesinos y campesinas, los derechos esenciales de las personas en condición de discapacidad y de los desplazados por razones del conflicto; los derechos fundamentales de las personas adultas mayores y de la población LGBTI (...). Valorando y exaltando que el eje central de la paz es impulsar la presencia y la acción eficaz del Estado en todo el territorio nacional, en especial en múltiples regiones doblegadas hoy por el abandono, por la carencia de una función pública eficaz, y por los efectos del mismo conflicto armado interno" (...), (Acuerdo Final para la Terminación del Conflicto y la Construcción de una Paz Estable y Duradera, págs. 2 y 3 ).

Es decir, los derechos fundamentales presentes en la educación, también tienen especial atención en el Acuerdo de Paz (2016). De igual manera:

El enfoque territorial del Acuerdo supone reconocer y tener en cuenta las necesidades, características y particularidades económicas, culturales y sociales de los territorios y las comunidades, garantizando la sostenibilidad socio-ambiental...La implementación se hará desde las regiones y territorios y con la participación de las autoridades territoriales y los diferentes sectores de la sociedad", (Acuerdo Final para la Terminación del Conflicto y la Construcción de una Paz Estable y Duradera, p. 6).

Estos dos enfoques, derechos fundamentales a la educación y territorialidad, sitúan en un marco de responsabilidades a las autoridades locales y de compromisos a las comunidades y a la academia que no se puede eludir, para lo cual será menester analizar y revisar la realidad de la precariedad de nuestro sistema educativo. 


\section{Debates conceptuales}

En el primer punto del Acuerdo Final de Paz (2016), "Hacia un Nuevo Campo Colombiano: Reforma Integral", se reconoce el acceso a la tierra como condición para la transformación del campo, también se señala que deben establecerse planes nacionales financiados y promovidos por el Estado para la prestación de servicios públicos como la educación, salud, recreación, alimentación, nutrición, etc., que generen calidad de vida a la población rural.

Dentro de estos Planes Nacionales para la Reforma Integral, cuyo objetivo central es la superación de la pobreza y la desigualdad, en el capítulo desarrollo social, se manifiesta, en el tema:

Educación rural: con el propósito de brindar atención integral a la primera infancia, garantizar la cobertura, la calidad y la pertinencia de la educación y erradicar el analfabetismo en las áreas rurales, así como promover la permanencia productiva de los y las jóvenes en el campo, y acercar las instituciones académicas regionales a la construcción del desarrollo rural, el gobierno nacional creará e implementará el Plan Especial de Educación Rural.

Para el desarrollo del Plan se tendrán en cuenta los siguientes criterios:

- La cobertura universal con atención integral a la primera infancia.

- Modelos flexibles de educación preescolar, básica y media, que se adapten a las necesidades de las comunidades y del medio rural, con un enfoque diferencial.

- La construcción, reconstrucción, mejoramiento y adecuación de la infraestructura educativa rural, incluyendo la disponibilidad y permanencia de personal docente calificado y el acceso a tecnologías de información.

- La garantía de la gratuidad educativa para educación preescolar, básica y media.

- El mejoramiento de las condiciones para el acceso y la permanencia en el sistema educativo de niños, niñas y adolescentes a través de un acceso gratuito a útiles, textos, alimentación escolar y transporte.

- La oferta de programas e infraestructura de recreación, cultura y deporte.

- La incorporación de la formación técnica agropecuaria en la educación media (décimo y once).

- La disponibilidad de becas con créditos condonables para el acceso de hombres y mujeres rurales más pobres a servicios de capacitación técnica, tecnológica y universitaria que incluya, cuando sea pertinente, apoyos a la manutención.

- La promoción de la formación profesional de las mujeres en disciplinas no tradicionales para ellas.

- La implementación de un programa especial para la eliminación del analfabetismo rural.

- El fortalecimiento y la promoción de la investigación, la innovación y el desarrollo científico y tecnológico para el sector agropecuario, en áreas como agroecología, biotecnología, suelos, etc.

- Incremento progresivo de los cupos técnicos, tecnológicos y universitarios en las zonas rurales, con acceso equitativo para hombres y mujeres, incluyendo personas en condición de discapacidad. Se tomarán medidas especiales para incentivar el acceso y permanencia de las mujeres rurales.

- Promover la ampliación de oferta y la capacitación técnica, tecnológica y universitaria en áreas relacionadas con el desarrollo 
rural. (Acuerdo final para la terminación del conflicto y la construcción de una paz estable y duradera, pp. 26 -27).

Planes y criterios que no solo son aplicables a la educación rural, sino a la educación que se brinda en las ciudades y municipios de la Región Caribe, a veces en condiciones de mayor marginalidad social que en el campo. Idea que se refuerza en Montes, Manning y Gamboa (2015).

La calidad de vida de los habitantes de la Región Caribe se ha venido deteriorando, según algunos indicadores, la tasa de incidencia de Pobreza Multidimensional (Índice IPM, salud, educación, empleo, vivienda, servicios públicos) fue de un $26.4 \%$, el segundo peor registro, solo superado por la Región Pacifica con un $33.2 \%$ y muy distante de la media nacional $(17.8 \%)$. De acuerdo con la información de la Gran Encuesta Integrada de Hogares (GEIH) del DANE, La tasa de desempleo para la Región Caribe fue de $8.9 \%$ en 2016 y la tasa de ocupación fue de 54.7\% para el mismo año, porcentajes menores en relación con las regiones centrales (Bogotá) y Oriental con un 64\% y $58,4 \%$ respectivamente, (Departamento Nacional de Estadísticas-DANE. Pobreza Monetaria y Multidimensional en Colombia 2016).

Esta precaria situación está vinculada a la poca capacidad de generación de ingresos por parte de los habitantes de la Región Caribe y es directamente proporcional a los bajos indicadores en el capital humano, trayendo como consecuencia que el Índice de Desarrollo $\mathrm{Hu}$ mano (IDH) medido por la longevidad de los habitantes, el acceso al saber y el bienestar material (0.645), se encuentre muy por debajo del promedio nacional (0.727), (Informe sobre Desarrollo Humano 2016. Panorama General. Desarrollo humano para todos. PNUD 2017).

\section{Metodología}

Esta investigación responde a una concepción epistemológica cualitativo-interpretativa de carácter descriptivo en la medida que se pretende identificar, entender y explicar la situación planteada con la realidad de la educación rural en Colombia frente a lo previsto en el Acuerdo de Paz (2016), como lo expresara (Colás, Buendía y Hernández (2009) "La finalidad de la investigación científica será comprender los fenómenos educativos, lográndose a través del análisis de las percepciones e interpretaciones de los sujetos que intervienen en la acción educativa" (p. 98).

Se utiliza el análisis documental de cifras y estadísticas, así como la experiencia directa de los actores involucrados en este sector rural del sistema educativo. Los datos cualitativos proporcionaran gran cantidad de significados diversos y revelaran distintas formas de comunicación, comportamiento, símbolos o artefactos culturales entre los actores de la investigación, Gibbs (2012) y eso es precisamente lo que se quiere conocer a fin lograr una aproximación a la realidad que se vive en las instituciones educativas frente a su contexto socioeconómico.

\section{Análisis de los resultados}

Consecuencia de esta realidad son los resultados en el sector educativo, existe una tasa de analfabetismo, del $32.6 \%$ en La Guajira la más alta en el país, muy superior al nivel nacional con un 9.7\%, siendo en el sector rural nacional $21.4 \%$, frente al urbano nacional con un $6.6 \%$ y una tasa de cobertura en educación básica y media por debajo del promedio del país (96.7\% y 43.8\%), frente a una cobertura bruta nacional de $98.35 \%$ (DANE/ MEN, 2015).

La Ley General de Educación (1994) define "la educación como un proceso de formación perma- 
nente, personal, cultural y social que se fundamenta en una concepción integral de la persona humana, de su dignidad, de sus derechos y de sus deberes", a su vez señala la obligación de promover un servicio de educación campesina y rural, formal, no formal e informal el cual comprenderá la formación técnica en las áreas agrícolas, pecuarias, pesqueras, forestales y agroindustriales, de igual manera implementar proyectos institucionales de educación campesina, (Ley 115 de Febrero 8 de 1994, artículos 1, 64 y 65), en consonancia con la Constitución Política de Colombia (1991, artículos 64 y 65) que considera un deber del Estado promover el acceso a los servicios educativos con el fin de mejorar el ingreso y la calidad de vida de los campesinos, así como garantizar la producción de alimentos.

Desde el año 2002 el Gobierno de Colombia viene promoviendo el Proyecto de Educación Rural a través del Programa de Fortalecimiento de la Cobertura con Calidad para el Sector Educativo Rural, Fase I y II (PER), con el cual se diseñaron e implementaron diversas estrategias pedagógicas dirigidas a docentes y estudiantes del campo, a fin garantizar el acceso y permanencia de los estudiantes, el fortalecimiento de las Secretarias de Educación territoriales, mejora de establecimientos educativos rurales, la promoción de los Proyectos Pedagógicos Productivos (PPP), dirigidos a la adquisición de competencias básicas y laborales con fundamento en experiencias reales de la vida del campo y que a su vez generaran una vinculación entre el sector educativo y el productivo.

Estrategias complementadas con los modelos de educación flexibles, como la aceleración del aprendizaje, escuela nueva, media rural, posprimaria, preescolar no escolarizado, entre otros.

Finalmente, dentro de este análisis normativo encontramos el Decreto Nacional 1075 de 2015 (Libro 2. Parte 3. Título 3. Capítulo 5. Sección 7, "Por medio del cual se expide el Decreto Único Regla- mentario del Sector Educación"), en donde se define que el ámbito de aplicación de la metodología Escuela Nueva se hará prioritariamente en la educación básica en todas las escuelas rurales del país, con el fin de mejorarla cualitativa y cuantitativamente, considerándose como rural todo establecimiento educativo situado en veredas, caseríos, corregimientos, inspecciones de policía, así como otras poblaciones dispersas que no estén en un centro poblado donde la alcaldía municipal tenga su sede administrativa.

Modelo pedagógico que se inició en Colombia en la década de los años 70 como respuesta a la poca escolaridad en el campo, debido a diferentes actividades agropecuarias en que participaban los niños y jóvenes, con este método multigrado se permitía al estudiante aprender al ritmo de su tiempo disponible, en pequeños grupos y con un docente que les guiara en las distintas áreas del conocimiento.

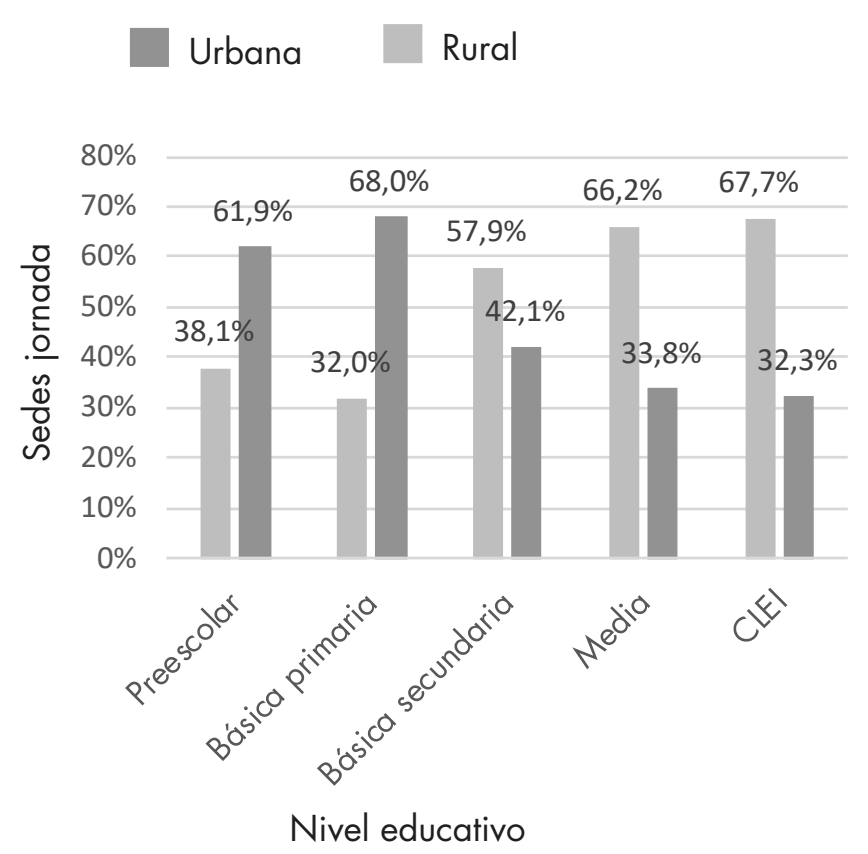

Gráfico 1. Distribución porcentual sedes por nivel educativo y zona 2016

Fuente: DANE. Boletín Técnico. Educación Formal 2016. Bogotá 18 de julio 2017. 
En el año lectivo 2016 los niveles educativos de preescolar y básica primaria fueron los que presentaron la mayor participación de sedes ubicadas en la zona rural con $61.9 \%$ y $68 \%$, respectivamente. Los niveles de básica secundaria, media y en la educación de adultos estructurada en ciclos lectivos especiales integrados-CLEI son los niveles con mayor participación ubicadas en la zona urbana, con $57.9 \%, 66.2 \%$ y $67.7 \%$ respectivamente.

De los alumnos matriculados en 2015, el 76.2\% fueron atendidos en establecimientos educativos en zona urbana y el $23.8 \%$ en la zona rural, en ese mismo año, el $72.8 \%$ de los docentes presta- ron sus servicios en instituciones educativas ubicadas en zona urbana y el $27.2 \%$ en sedes educativas ubicadas en zonas rurales, (Departamento Administrativo Nacional de Estadísticas-DANE. Investigación de Educación Formal 2015), en lo que respecta al Departamento de Bolívar por zona de atención en el año 2016 en la zona urbana fue de $140.453(58.55 \%)$ y en la zona rural 99.397 (41.44\%), lo que pone de presente el elevado número de estudiantes en el campo. (Ministerio de Educación Nacional de Colombia. Sistema Información de Matricula-SIMAT).

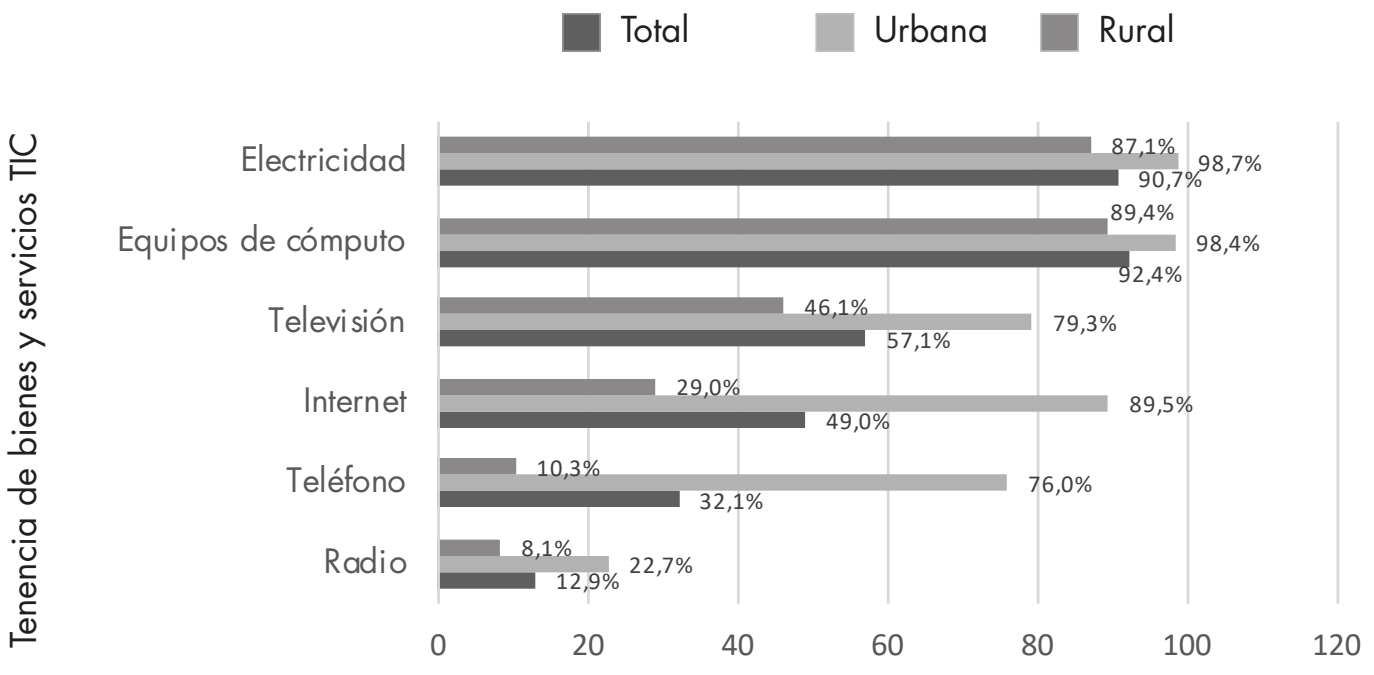

Gráfico 2. Sedes educativas con bienes y servicios TIC por zona 2016 Fuente: DANE. Boletín Técnico. Educación Formal 2016. Bogotá 18 de Julio 2017.

"De las 34.865 sedes educativas ubicadas en la zona rural, el 93,7\% (32.654) contaban con bienes y servicios TIC y el 6,3\% (2.211) no contaban con bienes y servicios TIC. De las 32.654 sedes, contaban con electricidad el $87,1 \%$, con equipos de cómputo el $89,4 \%$, con señal de televisión el $46,1 \%$, con internet el $29,0 \%$, con línea telefónica el 10,3\% y con señal de radio para fines educativos el $8,1 \%$.

De las 17.303 sedes educativas ubicadas en la zona urbana, $17.290(99,9 \%)$ de las sedes conta- ban con bienes y servicios TIC; con electricidad el $98,1 \%$, con equipos de cómputo el $98,4 \%$, con señal de televisión el 79,3\%, con internet el 89,5\%, con línea telefónica el 76,0\% y con señal de radio para fines educativos el 22,7\%. $13(0,1 \%)$ de las sedes no contaban con bienes y servicios TIC". (Departamento Nacional de Estadísticas-DANE. Investigación de Educación Formal 2016).

Nótese el desequilibrio porcentual en las herramientas TIC entre la zona rural y urbana demostrándonos una vez más las desigualdades que acarrea 
estudiar en el campo colombiano.

En Colombia el 75.5\% de los municipios son rurales, donde se concentra el $31.6 \%$ de la población, representando el 94.4\% del territorio. En el año 2016 las personas de 15 a 24 años residentes en las zonas urbanas tenían un promedio de 10.5 años de educación, mientras en que las zonas rurales este se reduce a un 8.4 años de educación y en relación con la tasa de asistencia, también se observan unos desbalances entre la educación rural y la urbana, para el rango de 5 a 16 años fue de $96.3 \%$ para las zonas urbanas y de $90.5 \%$ en zonas rurales, mientras que para el rango de edad entre los 17 a 24 años en las zonas urbanas fue de $43.1 \%$ y en las zonas rurales fue de $24.3 \%$, evidenciándose un descenso en este rango de edades que corresponde a los distintos niveles de la educación superior. (DANE. Encuesta Nacional de Calidad de Vida. ECV 2016). Todas estas situaciones de desbalances y desequilibrios traen como consecuencia unas bajas tasas de graduación, altas tasas de deserción, gran cantidad de jóvenes en extra edad con la correlativa disminución de acceso a la educación superior por parte de quienes vienen de una educación rural.

Es el momento preciso para darle un vuelco al sistema educativo a fin rescatar los principios y valores esenciales a la persona, la calidad educativa, la inclusión, la superación de la pobreza, amén de los criterios planteados en el Acuerdo Final de Paz (2016).

Es necesario adoptar una serie de estrategias y decisiones de política pública tales como vincular en la planta docente nacional los maestros y profesores necesarios con competencias pedagógicas para cada municipio en el área rural y urbana y que se les pague el salario de acuerdo al escalafón y competencias.

Terminar con la injusta proporción alumno/docente sobre todo en las zonas veredales; habrá que tenerse en cuenta las particularidades de los territorios; dotar de la infraestructura locativa necesaria, así como, de los instrumentos tecnológicos y pedagógicos indispensables para acceder al conocimiento global.

Garantizar de manera gratuita la seguridad alimentaria, los útiles, textos y uniformes escolares, el transporte escolar, llevar la educación superior en sus distintos niveles y con pertinencia curricular a las zonas rurales utilizando la infraestructura locativa de las instituciones de educación oficial en horarios nocturnos y fines de semana, con una real articulación entre la Educación Media y la Educación Superior, a través de los ciclos propedéuticos o de modelos flexibles que generen interés en los jóvenes y faciliten su permanencia en el sistema educativo.

En el caso particular del departamento de Bolívar, para solo mencionar un ejemplo, es necesario recuperar a los jóvenes "raspachines" de las hojas de coca o a quienes están vinculados con la minería ilegal y en los centros urbanos a los miembros de las pandillas juveniles, hoy llamados “jóvenes en riesgo", muchos de ellos provenientes de familias desplazadas por la violencia que azotaba su región, brindándoles oportunidades psicosociales, educativas y laborales, reparativas y acordes con la situación de violencia que vivió el campo como consecuencia del conflicto armado durante más de cincuenta años en el país.

Independientemente de cualquier condición ideológica, el Acuerdo Final de Paz (2016) debe ser el instrumento inspirador que guíe la reconciliación entre los colombianos y de ella por supuesto, cerrar la brecha social entre lo urbano y lo rural, en donde la educación sea el motor idóneo para la redefinición de un nuevo país con mejores condiciones de equidad y justicia social.

\section{Conclusiones}

- Es evidente que la ruralidad de Colombia ha generado limitaciones y desigualdades, especialmente en el sector educativo en donde los indicadores y estadísticas demuestran unas diferencias marcadas en términos negativos en relación con el sector urbano.

- A pesar que la normatividad constitucional, 
legal y los programas específicos amparan y promueven las oportunidades educativas y de desarrollo para el campesinado, estas no han sido suficientes para lograr avances significativos en términos de cobertura, calidad y mejoramiento para esta población ni para el área rural.

- El Acuerdo de Paz suscrito entre el Gobierno y las FARC (2016) contiene expresamente unos lineamientos para la educación rural dentro de un nuevo concepto de reforma integral que debe tener el campo colombiano dentro de los planes nacionales que deben ser promovidos y financiados por el Estado.

- Este escenario de paz es una excelente oportunidad para disminuir las desigualdades y terminar las iniquidades entre los sectores urbano y rural, cerrar las brechas y sanar las heridas de un conflicto armado que vivieron varias generaciones de colombianos a partir de un sistema educativo con calidad e incluyente.

\section{Referencias}

Colás B. M., Buendía E., L. y Hernández P., F. (2009). Competencias científicas para la realización de una tesis doctoral. España: Editorial Davinci.

Constitución Política de Colombia, julio (1991).

Departamento Nacional de Estadísticas. (2016). Encuesta Nacional de Calidad de Vida. ECV. Bogotá: DANE.
Departamento Nacional de Estadísticas. (2016). Investigación de Educación Formal. Bogotá: DANE.

Departamento Nacional de Estadísticas. (2016). Gran Encuesta Integrada de Hogares-GEIH. Bogotá: DANE.

Departamento Nacional de Estadísticas. (2016). Pobreza Monetaria y Multidimensional en Colombia Bogotá: DANE.

Gibbs, G., (2012). El análisis de datos cualitativos en Investigación Cualitativa. Madrid: Ediciones Morata, S.L.

Ley General de Educación. Ley 115 de febrero 8 de 1994.

Ministerio de Educación Nacional de Colombia. Decreto Nacional 1075 de (2015). Bogotá: MEN.

Ministerio de Educación Nacional. (2015). Programa de Fortalecimiento de la Cobertura con Calidad para el Sector Educativo Rural (PER). Bogotá: MEN.

Ministerio de Educación Nacional de Colombia. (2017). Sistema Información de Matrícula. Febrero 28/2017 Bogotá: SIMAT.

Montes, A. Manning, L. y Gamboa, A. (2015). Educación para el trabajo y el desarrollo humano. Un análisis desde la visión de los actores. Bogotá: ECOE Ediciones.

Presidencia de la República. (2016). Acuerdo final para la terminación del conflicto y la construcción de una paz estable y duradera. Bogotá.

Programa de las Naciones Unidas para el Desarrollo. (2017). Informe sobre Desarrollo Humano. Bogotá: PNUD. 